AperTO - Archivio Istituzionale Open Access dell'Università di Torino

\title{
Exploring the Organometallic Route to Molecular Spin Qubits: The [CpTi(cot)] Case
}

\section{This is a pre print version of the following article:}

Original Citation:

\section{Availability:}

This version is available http://hdl.handle.net/2318/1765157

since 2021-02-18T11:14:47Z

Published version:

DOI:10.1002/anie.202009634

Terms of use:

Open Access

Anyone can freely access the full text of works made available as "Open Access". Works made available under a Creative Commons license can be used according to the terms and conditions of said license. Use of all other works requires consent of the right holder (author or publisher) if not exempted from copyright protection by the applicable law. 


\section{The Organometallic Route to Highly Coherent Molecular Spin Qubits: the [CpTi(cot)] case}

Luana C. de Camargo, ${ }^{\S}$ Matteo Briganti, ${ }^{\S}$ Francielli S. Santana, Danilo Stinghen, Ronny R. Ribeiro, Giovana G. Nunes, Jaísa F. Soares, ${ }^{*}$ Enrico Salvadori, Mario Chiesa, ${ }^{\star}$ Stefano Benci, Renato Torre, Lorenzo Sorace, Federico Totti, and Roberta Sessoli*

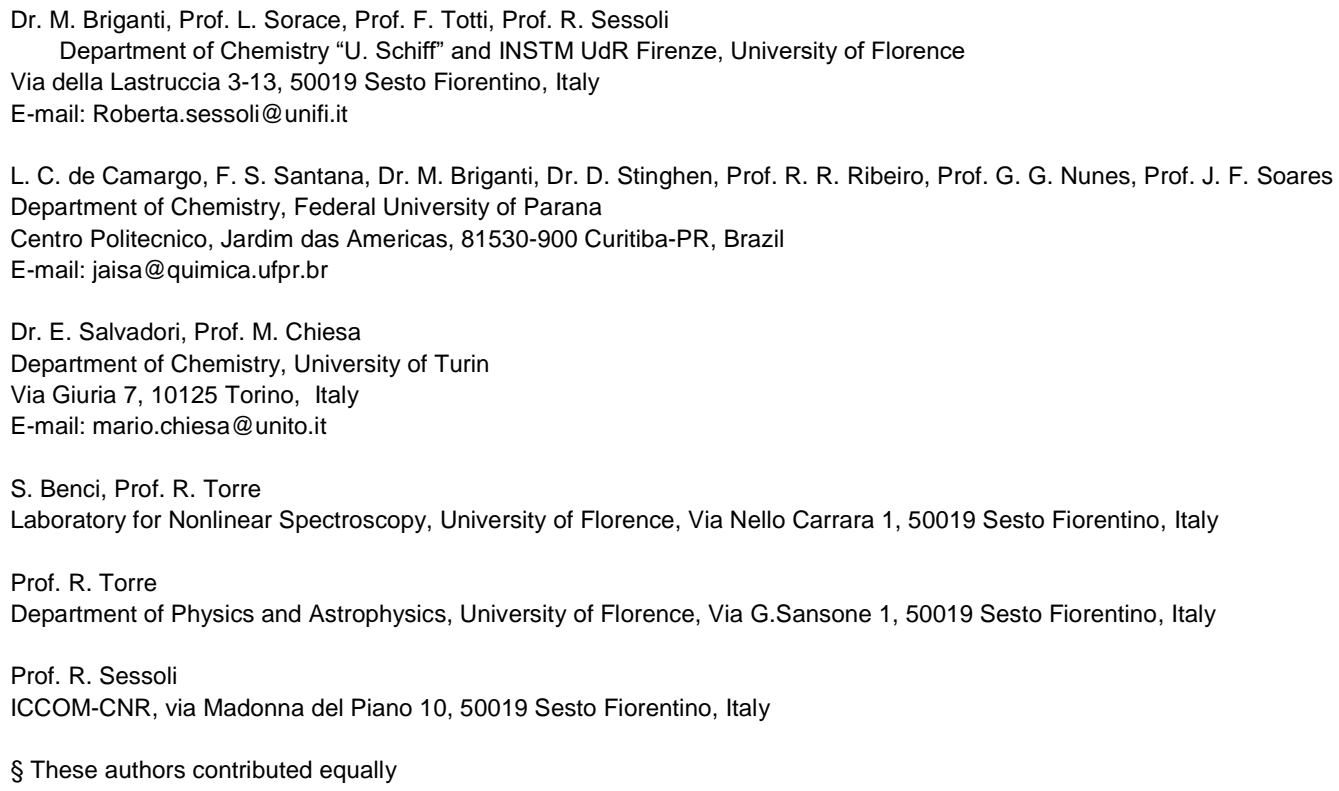


Abstract: The coherence time of the 17-electron, mixed sandwich complex [CpTi(cot)], ( $\eta^{8}$-cyclooctatetraene $)\left(\eta^{5}\right.$-cyclopentadienyl)titanium, was found to reach $34 \mu \mathrm{s}$ at $4.5 \mathrm{~K}$ in a frozen deuterated toluene solution. This result represents a remarkable coherence time for a highly protonated molecule. Ab initio calculations revealed that the low energy vibrational modes involving the rotation of the ligand rings have negligible spin-phonon coupling. At the same time, hydrogen nuclear spins are too strongly coupled with the titanium electronic spin $(S=1 / 2)$ to affect the electronic spin coherence negatively. This, however, is not the case for hydrogen atoms of neighboring molecules of the diamagnetic (16-electron) crystalline host [CpTi(cht)], $\left(\eta^{7}\right.$-cycloheptatrienyl)( $\eta^{5}$-cyclopentadienyl)titanium. The volatility of these neutral species makes them ideal candidates for single-qubit addressing on surface and quantum sensing in combination with scanning probe microscopies.

Organometallic sandwich complexes of paramagnetic lanthanide ions are currently the focus of great interest because of the observation that dysprosium(III) derivatives present magnetic hysteresis of molecular origin, i.e. Single-Molecule Magnet (SMM) behavior, above liquid nitrogen temperature. ${ }^{[1]}$ This outstanding performance results from a strongly axial crystal field. Equally relevant to the slow relaxation of the magnetization is the reduced spin-phonon coupling of the metal-ligand vibrational modes given the delocalized electron density on the ligand. ${ }^{[1 a, 2]}$ Concerning $3 \mathrm{~d}$ metal ions, ferrocinium complexes show slow relaxation of the magnetization in applied static fields. ${ }^{[3]}$ Additionally, the neutral nickelocene complex, bis $\left(\eta^{5}\right.$-cyclopentadienyl)nickel, characterized by the spin state $S=1$ with easy plane anisotropy, has revealed an outstanding capability to act as local magnetic sensor when adsorbed on the tip of a scanning tunnel microscope..$^{[4]}$

Sandwich complexes of early-transition metal ions such as $\mathrm{Ti}$ and $\mathrm{V}$ carrying one unpaired electron are also well known, but, to the best of our knowledge, their spin dynamics has not been reported. In our search for highly coherent evaporable magnetic molecules as potential molecular spin qubits, ${ }^{[5]}$ we identified in $[\mathrm{CpTi}(\cot )]$ (1 in Figure 1a), where $\mathrm{Cp}=\eta^{5}-\mathrm{C}_{5} \mathrm{H}_{5}$ and $\cot =\eta^{8}-\mathrm{C}_{8} \mathrm{H}_{8}$ are formally treated as mono- and di-anions respectively, a promising $S=1 / 2$ candidate. In 1 , the unpaired electron occupies an orbitally non-degenerate state and vibrations are not expected to be very efficient in promoting spin relaxation. The synthesis, ${ }^{[6]}$ crystal structure,$^{[7]}$ continuous-wave $(\mathrm{CW}) \mathrm{EPR}^{[8]}$, and ENDOR ${ }^{[9]}$ spectra were reported for $\mathbf{1}$ a few decades ago. Some relevant data are revisited and updated in the present work. Besides, we report here a pulsed EPR investigation combined with ab initio calculations to verify if the unique electronic and vibrational properties of this class of molecules are also beneficial to boost coherence time in $S=1 / 2$ molecules. We found that despite the very low energy of the rotational mode of the rings and the presence of many hydrogen atoms in the ligands, coherence time in frozen solution reaches values of tens of $\mu$ s that are remarkable for a hydrogen-rich molecule.

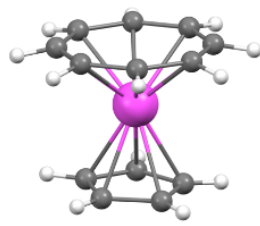

[CpTi(cot)], 1

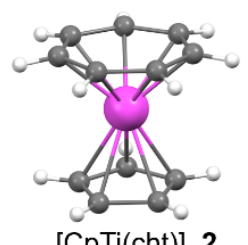

[CpTi(cht)], 2

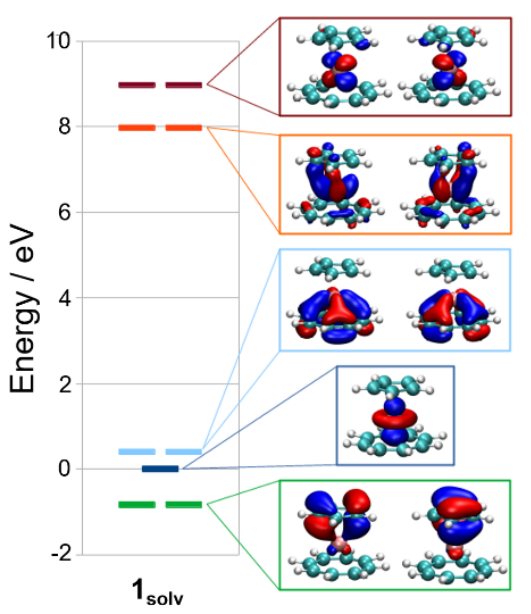

Figure 1. a) Molecular structure of the paramagnetic [CpTi(cot)] and of the diamagnetic [CpTi(cht)] complexes; b) Energies and wave-functions of the molecular orbitals of $[\mathrm{CpTi}(\mathrm{cot})]$ in the presence of implicit toluene solvent, computed by the Conductor-like Polarizable Continuum Model.

Synthesis of 1 was performed by a new route that comprises reaction of $\left[\mathrm{Cp}_{2} \mathrm{TiCl}_{2}\right]$ with $n$-butyllithium in the presence of excess of cyclooctatetraene. More details on these preparations and characterizations are provided as Supplementary Information (SI). After sublimation of the crude product at $120{ }^{\circ} \mathrm{C}$ and $10^{-3} \mathrm{~mm} \mathrm{Hg}$, green crystalline 1 was isolated in ca. $70-80 \%$ yield. X-ray data collection was performed at $100 \mathrm{~K}$ to improve accuracy, and, despite a previous report of a destructive phase transition below $200 \mathrm{~K},{ }^{[10]}$ the molecular and crystal structures solved for $\mathbf{1}$ did not differ significantly from those investigated at higher temperatures (Table S4) . A preliminary essay of dispersion in a diamagnetic crystalline matrix was performed by co-crystallizing $\mathbf{1}$ with [CpTi(cht)] (2, cht $=\eta^{7}-\mathrm{C}_{7} \mathrm{H}_{7}{ }^{3-}$, Fig.1a) in nominal 1:30 molar proportion (ca. 3 mol\%). Synthesis ${ }^{[11]}$ and crystal structure ${ }^{[12]}$ of 2 were also reported in the past and were again revisited here to confirm the identity of the product and the adequacy of the new synthetic route.

X-band $(v \approx 9.5 \mathrm{GHz})$ CW-EPR spectra of 1 dissolved in toluene $\left(1.0 \mathrm{mmol} \mathrm{L} \mathrm{L}^{-1}\right)$ at variable temperature are shown in Figure $2 \mathrm{a}$, while Q-band $(v \approx 33.8 \mathrm{GHz}$ ) CW and echo-detected field-swept (EDFS) EPR spectra of 1 in deuterated toluene at 5 $\mathrm{K}\left(0.5 \mathrm{mmol} \mathrm{L}^{-1}\right)$ are reported in Fig. $2 \mathrm{~b}$. The low-temperature fully-frozen spectra show the typical features of an axially anisotropic $S=1 / 2$ species with $g_{/ /} \approx g_{e}>g_{\perp}$, as expected when the SOMO has the main contribution from the non-bonding $\mathrm{dz}^{2}$ metal orbital. In contrast the powder EPR spectrum was reported to show a reversed pattern with $g_{/ /}<g_{\perp}<g_{e}$, due to the averaging, promoted by magnetic exchange, of $g$ components between molecules that have their $z$-axis orthogonal to each other. ${ }^{\left[{ }^{[8]}\right.}$ This is here confirmed by the EPR spectrum of $\mathbf{1}$ diluted in $\mathbf{2}$ (Fig. S6), which presents the same $g$ anisotropy of the frozen solution.

Main lines are flanked by the hyperfine components due to the different stable $\mathrm{Ti}$ isotopes $\left({ }^{46} \mathrm{Ti},{ }^{48} \mathrm{Ti},{ }^{50} \mathrm{Ti} l=0\right.$, natural abundance, n.ab.=87.15\%; ${ }^{47} \mathrm{Ti}, l=5 / 2$, n.ab. $=7.44 \% ;{ }^{49} \mathrm{Ti}, l=7 / 2$, n.ab.=5.41\%). ${ }^{1} \mathrm{H}$ super-hyperfine structures are only visible at intermediate temperatures (250-180 K), when the tumbling of the molecules averages the anisotropic components. At room temperature, such a super-hyperfine structure is partially lost due to line broadening. (see Fig. 2 and Fig. S4). 
Simulation of the spectra over the whole temperature range was performed by considering a temperature-dependent correlation time in the intermediate regime (more details in SI). ${ }^{[13]}$ The values of the spin Hamiltonian (SH) parameters are reported in Table 1 and compare well with previous reports ${ }^{[8,9 b]}$ including detailed ENDOR investigations. ${ }^{[9 a]}$

Figure 2. Field-swept EPR spectra of toluene solutions of 1. In the left panel, experimental X-band CW-EPR spectra between $77 \mathrm{~K}$ and RT are reported together with simulation as thin black lines (see text and SI for details). In the right panel, experimental and simulated (thin black lines) frozen solution spectra at $T=4.5 \mathrm{~K}$ and different frequencies are plotted on $\mathrm{g}$-scale to ease comparison.

Table 1. Spin Hamiltonian parameters extracted from EPR spectra compared with computed data assuming the $\mathrm{X}$-ray molecular structure and optimizing the structure in the presence of toluene as implicit solvent. Hyperfine and quadrupolar couplings are given in $\mathrm{MHz}$.

\begin{tabular}{|c|c|c|c|c|}
\hline & Exp. ${ }^{[a]}$ & Exp. $^{[b]}$ & 1x-ray & $1_{\text {solv }}^{[c]}$ \\
\hline$g_{x}$ & $1.972(3)$ & $1.972(3)$ & 1.972 & 1.971 \\
\hline$g_{y}$ & $1.972(3)$ & $1.972(3)$ & 1.973 & 1.972 \\
\hline$g_{z}$ & $2.001(1)$ & $2.000(8)$ & 2.002 & 2.002 \\
\hline$A_{\text {iso }}\left({ }^{1} \mathrm{H}-\mathrm{Cp}\right)$ & $4.1(3)$ & 4.09 & +2.7 & +3.4 \\
\hline$A_{\text {iso }}\left({ }^{1} \mathrm{H}-\cot \right)$ & $8.8(3)$ & 8.78 & +6.2 & +7.4 \\
\hline$A_{x}\left({ }^{47} \mathrm{Ti}\right)$ & $52.4(8)^{[d]}$ & $52.60(5)^{[\mathrm{e}]}$ & +52 & +53.8 \\
\hline$A_{y}\left({ }^{47} \mathrm{Ti}\right)$ & $52.4(8)^{[d]}$ & $52.60(5)^{[e]}$ & +53 & +54.1 \\
\hline$A_{z}\left({ }^{47} \mathrm{Ti}\right)$ & ca. 5 & ca. 10 & -2.6 & -0.6 \\
\hline$P_{I I}\left({ }^{47} \mathrm{Ti}\right)^{[f]}$ & - & $-2.30(2)$ & -1.81 & - \\
\hline$P_{I I}\left({ }^{49} \mathrm{Ti}\right)^{[\mathrm{f}]}$ & - & $-0.90(2)$ & -0.71 & - \\
\hline
\end{tabular}
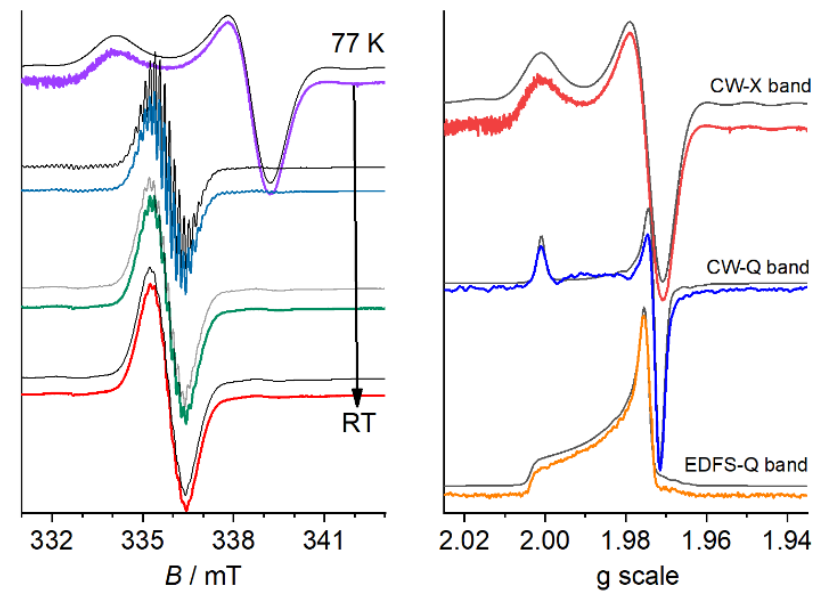

[a] This work. [b] From ENDOR studies. ${ }^{[9 a]}$ [c] The solvent was considered by CPCM model. [d] Values for ${ }^{49}$ Ti can be obtained by appropriate rescaling. See SI for more details. [e] Average value $\left({ }^{47} \mathrm{Ti}, 52.80 ;{ }^{49} \mathrm{Ti}, 52.40 \mathrm{MHz}\right)$. [f] $H_{Q S}=P /\left[I^{2}-1 / 3(I+1)\right]$.

As a first necessary step for the rationalization of the spin dynamics in this molecule, we calculated the static $\mathrm{SH}$ parameters using ORCA 4.0 quantum chemistry package. ${ }^{[14]}$ Density Functional Theory (DFT) calculations of the electronic structure with B2PLYP ${ }^{[15]}$ double hybrid functional were performed on three different geometries of the isolated molecule: i) the X-ray structure

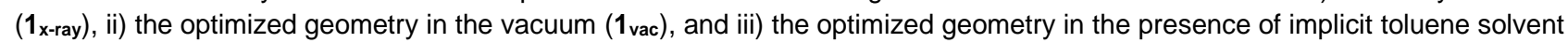

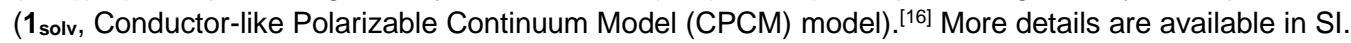

The optimized structure of $[\mathrm{CpTi}(\mathrm{cot})]$ in vacuum (1 $\mathbf{v a c})$ is in excellent agreement with the experimental findings, i.e., C-C bonds and the distance between $\mathrm{Ti}$ and the centroids of the two rings show a deviation smaller than $1.5 \%$ and $0.5 \%$, respectively. Only a moderate increase in $\mathrm{C}-\mathrm{H}$ bond length is observed (Table S7). Optimizing the structure in the solvent (1 $\mathbf{\text { solv}}$ ) provides similar geometrical parameters. The energy barrier for the rotation of the two rings around the z-axis, estimated by rigidly rotating the $\mathrm{Cp}$ ring in the optimized structure with solvent, is found to be very small, ca. $40 \mathrm{meV}\left(350 \mathrm{~cm}^{-1}\right)$.

The electronic structure of [CpTi(cot)] in $\mathbf{1}_{\text {xray }}, \mathbf{1}_{\text {vac }}$ and $\mathbf{1}_{\text {solv }}$ (see Figure $\mathbf{1 b}$ for $\mathbf{1}_{\text {solv, }}$, and Fig. S15 for comparison) is characterized by the splitting of the $d$ orbitals in three sets: non-bonding, bonding, and anti-bonding. The latter two arise from the interaction of metal $d$ orbitals with the $\pi$ systems of the rings. If we take the energy of the non-bonding SOMO $d_{z 2}$ as reference, the two bonding sets lie at $-0.8\left(d_{x z} / d_{y z}\right)$ and $0.3\left(d_{x y} / d_{x 2-y 2}\right)$ eV respectively, while the antibonding sets lie at $7.9\left(d_{x y} / d_{x 2-y 2}\right)$ eV and $9.0 \mathrm{eV}\left(\mathrm{d}_{\mathrm{xz}} / \mathrm{d}_{\mathrm{yz}}\right)$

Interestingly, in [CpTi(cot)] the interactions between the Ti d orbitals and the $\pi$ systems of the two ligands are quite selective, as reported earlier for $[\mathrm{CpM}(\mathrm{cht})]$ complexes, $\mathrm{M}=\mathrm{Cr}$, V, Ti. ${ }^{[17]}$ Indeed, the $\mathrm{d}_{\mathrm{xz}}$ and $\mathrm{d}_{\mathrm{yz}}$ orbitals $\left(\mathrm{e}_{1} ; \mathrm{C}_{\infty \mathrm{v}}\right.$ approximation) interact selectively with the Cp, while the $d_{x y}$ and $d_{x 2-y 2}\left(e_{2}\right)$ orbitals show selective interactions with the cot $\pi$ system (see Fig 1b). The electric dipole moment is computed to be 0.98 D.

The calculated $\mathrm{SH}$ parameters for $\mathbf{1}_{\mathrm{vac}}$ and $\mathbf{1}_{\text {solv }}$ are also gathered in Table 1. The agreement with experimental data is excellent and proves the reliability of the computational protocol. The discrepancy between the experimental and calculated values for $\left.A_{z}{ }^{47} \mathrm{Ti}\right)$ has to be considered in the light of the large uncertainty on the experimental values. ${ }^{[9 a]}$ More subtle details, such as the anisotropic contributions to the ${ }^{1} \mathrm{H}$ hyperfine interaction, are also well reproduced (Table S10).

Spin-lattice relaxation rates were investigated by pulsed EPR, using inversion-recovery experiments, for $1 \mathrm{mmol} \mathrm{L^{-1 }}$ (4.5-150 K) and $0.5 \mathrm{mmol} \mathrm{L}^{-1}$ solutions (temperature ranges

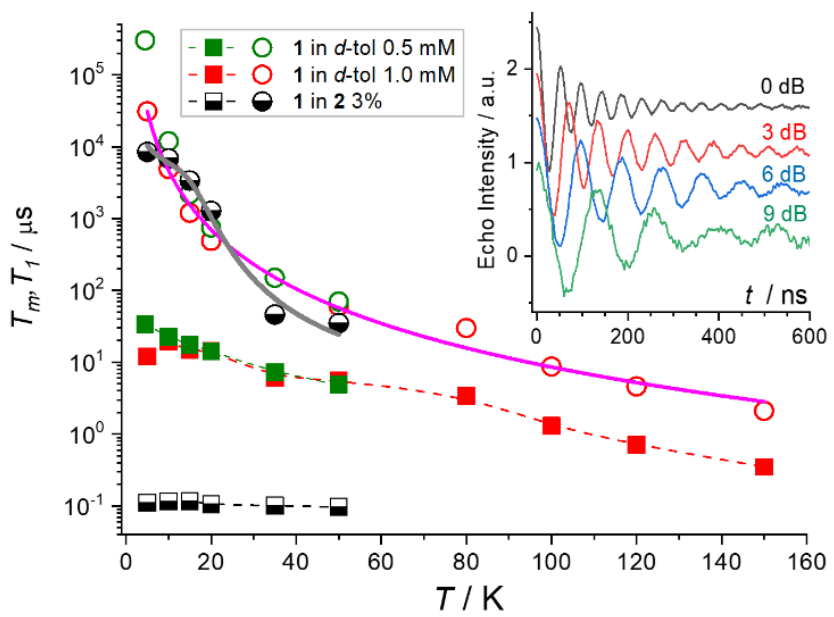
of $4.5-150 \mathrm{~K}$ and $4.5-50 \mathrm{~K}$ respectively). More details are available in the SI. The resulting saturation-recovery traces recorded 
at the $g_{\perp}$ resonance field (Fig. S9) were fitted with a standard stretched exponential equation (stretching parameter in the range $0.68-0.93)$, and the extracted $T_{1}$ values are reported in Figure 3 . The temperature dependence was simulated over the entire investigated temperature range assuming a power-law $T^{-n}$, with $n=2.7(1)$ (solid pink line in Fig. 3). There is no evidence of the crossover to the direct regime with $n \sim 1$ usually observed at low temperature for $S=1 / 2$ systems. ${ }^{[18]}$ The difference observed for the two similar concentrations probably depends on slightly different freezing times that affect the organization of the solvent around the molecules of $\mathbf{1}$ (vide infra). ${ }^{\text {9b] }}$

Hahn-echo decay experiments were performed in solution to investigate the coherence time of 1 . Deuterated toluene was employed to reduce the solvent contribution to decoherence. The phase memory times, $T_{m}$, extracted from the simulation of the decay traces (Fig. S9) provide consistent behavior either using a stretched exponential or a bi-exponential decay. Long $T_{m}$ values, above $10 \mu \mathrm{s}$, are observed below $20 \mathrm{~K}$ as shown in Figure 3 (solid squares). This is a remarkably robust coherence for a hydrogenrich molecule, exceeding or comparable to those observed for nuclear spin-free ligands in frozen ${ }^{[19]}$ and solid solutions, ${ }^{[20]}$ respectively.

Nutation experiments were also performed at $80 \mathrm{~K}$ (inset of Fig. 3), and Rabi oscillation frequencies were found to scale linearly with the microwave magnetic field (Fig. S10), indicating that coherent spin manipulation is feasible.

The moderate detrimental effect of hydrogen nuclear spins on $T_{m}$ is not unexpected and indeed was one of the motivations of our study. It is well known that moderately-coupled nuclear spins, i.e., those beyond the diffusion barrier ${ }^{[21]}$ (experimentally estimated around $0.6 \mathrm{~nm}$ ), have the largest decoherence efficiency. ${ }^{[22]}$ In the present case, the hydrogen atoms of the two ligands are much closer to the Ti spin $(\mathrm{ca} .0 .3 \mathrm{~nm})$ and strongly coupled. Their contribution to decoherence is then limited, although the rotation of the rings is not fully hindered. To verify this hypothesis, we also investigated the spin dynamics of a nominal $3 \%$ solid solution of 1 in $\mathbf{2}$ by pulsed EPR (see SI). The extracted characteristic times are reported in Figure 3 and evidence a striking decrease in $T_{m}$ to $0.1 \mu \mathrm{s}$, virtually temperature independent. This suggests that decoherence induced by the hydrogen nuclear spins of neighboring diamagnetic molecules of 2 is very efficient.

Figure 3. Temperature dependence of $T_{1}$ and $T_{m}$ extracted from Q-band pulsed-EPR experiments performed for deuterated toluene frozen solutions of 1 at two concentrations, and for a solid solution of $\mathbf{1}$ in $\mathbf{2}$. The solid lines represent the best-fit curves reproducing $T_{1}$ (see text). Broken lines are only a guide to the eye. The inset presents the normalized echo intensity in nutation experiments for the $0.5 \mathrm{mmol} \mathrm{L}^{-1}$ solution of 1 at $T=80 \mathrm{~K}$.

It is interesting to notice that comparable spin-lattice relaxation times $\left(T_{1}\right)$ are observed for frozen and solid solutions, though with different temperature dependence. Attempts to reproduce the data of the solid solution reported in Figure 3 with a power law, as for the frozen solution, failed completely. An acceptable agreement was found by adding an exponential contribution to the relaxation rate, as suggested in the case of active optical vibration modes,

$$
T_{1}^{-1}=a T^{n}+\operatorname{bexp}\left(-\frac{\Delta}{k_{B} T}\right)
$$

with $\Delta=129(25) \mathrm{K}\left(90 \mathrm{~cm}^{-1}\right)$. Though poorly defined, the small $\mathrm{n}=0.4(8)$ value is similar to what observed for other $S=1 / 2$ systems in solid solutions. ${ }^{[23]}$

A deeper understanding of the peculiar quantum spin dynamics of this organometallic $S=1 / 2$ system requires to investigate in detail its vibrational properties and their influence on the spin dynamics, a point which we tackle in the following, both experimentally and theoretically.

An early report on the vibrational properties of 1 listed two weak IR-active bands at 61 and $143 \mathrm{~cm}^{-1}$, respectively, while the first intense band was reported at $377 \mathrm{~cm}^{-1}$. $^{[2]}$ We performed time-domain $\mathrm{THz}$ spectroscopy experiments on a polycrystalline sample of 1 dispersed in high-density polyethylene to detect the presence of low energy modes. More details on such technique are reported in SI. Figure 4 presents the spectra recorded at different temperatures between 20 and $400 \mathrm{~cm}^{-1}$. Early findings were fully confirmed, and no significant absorptions were observed below the one at $75 \mathrm{~cm}^{-1}$. A small blue-shift on decreasing the temperature was observed for the low-energy vibration modes, as found for other complexes. ${ }^{[23 a, 25]}$ 
A theoretical analysis of the low-energy vibrational modes was performed, assuming the optimized geometry of $\mathbf{1}$ both in vacuum, $\mathbf{1}_{\mathrm{vac}}$, and in the presence of toluene, $\mathbf{1}_{\mathrm{sol}}$ (see SI for more details). Table 2 reports the computed vibration frequencies for the three lowest energy modes, while the associated displacements vectors are shown in the inset of Figure 4 . Noticeable is the extremely low frequency of the ring rotations for the molecule in vacuum $\left(8 \mathrm{~cm}^{-1}\right)$, in agreement with the small energy barrier preventing free rotation. The inclusion of the solvent has a dramatic effect on this vibration, whose frequency almost triplicates. The two quasi-degenerate modes just above $100 \mathrm{~cm}^{-1}$ were found to correspond to two orthogonal ring-metal-ring bending motions, and are weakly sensitive to the environment. These results support a previous tentative assignment of the Terahertz spectra (see Figure 4). ${ }^{[24]}$

Figure 4. Absorption of $\mathrm{THz}$ radiation of 1 dispersed in high-density polyethylene at different temperatures. In the inset, the displacements vectors computed for the first three lowest-energy vibration modes are reported and here tentatively assigned to the observed IR bands.

The spin-vibrational coupling was computed for the low energy vibrations to correlate vibrational properties with spin dynamics. Given that the spin dynamics was investigated at moderately high magnetic field $(1.2 \mathrm{~T})$ and the most abundant $\mathrm{Ti}$

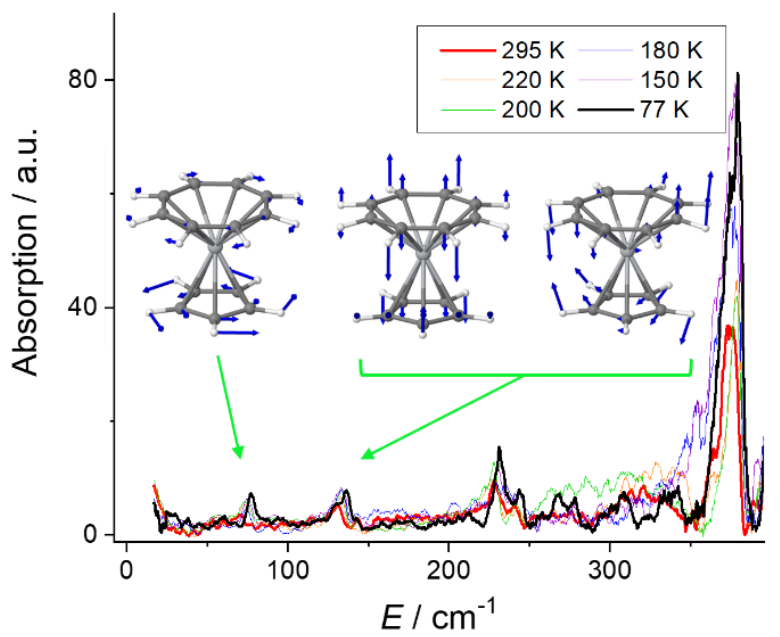
isotopes have $l=0$, modulation of the $\mathbf{g}$ components is expected to prevail over hyperfine interactions in promoting spin-lattice relaxation. We evaluated the effect of the geometrical distortions induced by each vibration on the $\mathbf{g}$ tensor of the molecule using a well-established procedure. ${ }^{[1 a, 25-26]}$ Briefly, the molecule was distorted along each normal mode, the nine components of the $\mathbf{g}$ tensor were computed, and their variation fitted with a quadratic polynomial function. Finally, the square of the nine linear term coefficients were summed up to give the relative spin-vibrational coupling coefficient reported in Table 2.

In both isolated and solvated molecules the two vibrations above $100 \mathrm{~cm}^{-1}$ are computed to be 4-8 times more efficient in the relaxation process than the low-energy rings rotation mode. This finding agrees with the fact that the SOMO is a $d_{z 2}$ orbital, and its interaction with the $\pi$ systems is not affected by the lowest vibration due to its symmetry.

Table 2. Computed frequencies $\left(\mathrm{cm}^{-1}\right)$ and spin-phonon coupling coefficients for the three lowest-energy vibration modes in 1, assuming a molecule in the vacuum or in the presence of implicit toluene.

\begin{tabular}{l|ll|ll}
\hline \multirow{2}{*}{$\begin{array}{l}\text { Vibration } \\
\text { mode }\end{array}$} & \multicolumn{2}{|c|}{$\mathbf{1}_{\text {vac }}$} & \multicolumn{2}{c}{$\mathbf{1}_{\text {solv }}$} \\
\cline { 2 - 5 } & Freq. & Sp-Ph Coupl & Freq. & Sp-Ph Coupl \\
\hline 1 & 8 & $1.3 \times 10^{-8}$ & 22 & $7.8 \times 10^{-9}$ \\
2 & 107 & $6.4 \times 10^{-8}$ & 111 & $5.3 \times 10^{-8}$ \\
3 & 111 & $4.3 \times 10^{-8}$ & 115 & $4.5 \times 10^{-8}$ \\
\hline
\end{tabular}

It is interesting to correlate our vibrational analysis with the temperature dependence of $T_{1}$ reported in Figure 3 . The rapid increase of $T_{1}$ at low temperature with a temperature exponent close to 3 suggests that multi-phonon mechanisms involving optical modes, e.g. Raman, dominate over the direct process involving acoustic modes. The fact that a unique exponent is observed for the deuterated toluene solution in the whole temperature range could be due to a wide distribution of frequencies for these modes as a result of the different environments generated by the solvent molecules. It is interesting to notice that for the solid solution of 1 in 2 the temperature dependence of $T_{1}$ is significantly different; in this case, one optical mode seems to dominate. The activation energy extracted from $T_{1}$ of the solid solution falls in between the two lowest-energy, experimentally detected, vibrational modes for 1. Unfortunately, a direct comparison between $\mathrm{THz}$ spectra of $\mathbf{1}$ and spin dynamics of the solid solution is hampered by the fact that, despite the same space group and similar unit cell volumes, the diamagnetic matrix $\mathbf{2}$ is not strictly isostructural with $\mathbf{1}$ (details in the $\mathrm{SI}$ ). Moreover, optical spectroscopy gives only access to vibration at the $\Gamma$ points, but the entire Brillouin zone is contributing, requiring a more detailed analysis. ${ }^{[27]}$

To conclude, this simple $S=1 / 2$ organometallic molecule presents very appealing spin coherence properties, despite a large number of hydrogen atoms and the mobility of the rings. This is due to the strong hyperfine coupling of the electronic and nuclear spins on the titanium and hydrogen atoms, respectively. For this reason, dilution in an isostructural diamagnetic matrix is not an appropriate way to improve coherence time, since it introduces dipolar interactions to a large number of ${ }^{1} \mathrm{H}$ nuclear spins of neighboring molecules. On the other hand, $\mathbf{1}$ is a neutral and easily sublimable molecule with longer $T_{m}$ than those of vanadyl or copper(II) phthalocyanine and porphyrin molecules. It appears, therefore, an ideal candidate to act as a coherent spin sensor at the single-molecule level, similarly to what was recently achieved with [ $\left.\mathrm{NiCp}_{2}\right]$ for non-coherent nanoscale magnetic sensing. ${ }^{[4 \mathrm{~b}]}$

Although the presence of very low energy vibrations represents a possible weakness of these small sandwich molecules, our theoretical analysis indicates that these vibrational modes have a relatively weak spin-phonon coupling, and therefore $T_{1}$ 
remains long enough not to affect $T_{m}$ over a reasonable temperature range. Under this respect [CpTi(cot)] may be seen as an $S=1 / 2$ analogue of what dysprosium(III) metallocene cations currently represent in the field of SMMs. ${ }^{[12-d, 2]}$

We can foresee many different avenues to improve the coherence properties of this type of molecules. The introduction of an interannular link, as in silatrovacenophanes, ${ }^{[28]}$ will inhibit the rotations of the rings, allowing disentangling different contributions to the spin relaxation and, likely, further slowing down the spin dynamics. Electric field control can also be envisaged for this acentric and polar molecule, ${ }^{[29]}$ as well as single spin coherent manipulation with a scanning tunnel microscope, as recently achieved for $\mathrm{Ti}$ adatoms on $\mathrm{MgO} .{ }^{[30]}$ Noticeable is also the non-negligible quadrupolar nuclear splitting for the ${ }^{47} \mathrm{Ti}$ isotope that could allow implementation of error correction protocols by using the metal hyperfine levels. ${ }^{[31]}$

\section{Acknowledgements}

This work has been supported by Italian MIUR (Project PRIN 2015-HYFSRT and Progetto Dipartimenti di Eccellenza 2018-2022, ref B96C1700020008), by the EU Commission through the QuantERA Project SUMO and the FETOPEN project FATMOLS (GA 862893), and by the Brazilian CNPq (Project 308426/2016-9) and CAPES (PROEX and PrInt/CAPES-UFPR, Finance Code 001). The computing resources and the related technical support used for this work have been provided by CRESCO/ENEAGRID High Performance Computing infrastructure ${ }^{[32]}$ and its staff.

Keywords: Titanium • Metallocene complexes • Electron Paramagnetic Resonance • Quantum Coherence • DFT

[1] a) C. A. P. Goodwin, F. Ortu, D. Reta, N. F. Chilton, D. P. Mills, Nature 2017, 548, 439; b) G. Fu-Sheng, D. B. M., C. Yan-Cong, T. Ming-Liang, M. Akseli, L. R. A., Angew. Chem. Int. Ed. 2017, 56, 11445-11449; c) F.-S. Guo, B. M. Day, Y.-C. Chen, M.-L. Tong, A. Mansikkamäki, R. A. Layfield, Science 2018, 362, 1400-1403; d) K. Randall McClain, C. A. Gould, K. Chakarawet, S. J. Teat, T. J. Groshens, J. R. Long, B. G. Harvey, Chem. Sci. 2018, 9, 8492-8503; e) C. A. Gould, K. R. McClain, J. M. Yu, T. J. Groshens, F. Furche, B. G. Harvey, J. R. Long, J. Am. Chem. Soc. 2019, 141, $12967-12973$.

[2] L. Escalera-Moreno, J. J. Baldoví, A. Gaita-Ariño, E. Coronado, Chem. Sci. 2020, 11, 1593-1598.

[3] M. Ding, A. K. Hickey, M. Pink, J. Telser, D. L. Tierney, M. Amoza, M. Rouzières, T. J. Ozumerzifon, W. A. Hoffert, M. P. Shores, E. Ruiz, R. Clérac, J. M. Smith, Chem. Eur. J. 2019, 25, 10625-10632.

[4] a) B. Verlhac, N. Bachellier, L. Garnier, M. Ormaza, P. Abufager, R. Robles, M. L. Bocquet, M. Ternes, N. Lorente, L. Limot, Science 2019, 366, 623627; b) G. Czap, P. J. Wagner, F. Xue, L. Gu, J. Li, J. Yao, R. Q. Wu, W. Ho, Science 2019, 364, 670-673; c) M. Ormaza, N. Bachellier, M. N. Faraggi, B. Verlhac, P. Abufager, P. Ohresser, L. Joly, M. Romeo, F. Scheurer, M. L. Bocquet, N. Lorente, L. Limot, Nano Lett. 2017, 17, 1877-1882.

[5] a) J. Ferrando-Soria, E. M. Pineda, A. Chiesa, A. Fernandez, S. A. Magee, S. Carretta, P. Santini, I. J. Vitorica-Yrezabal, F. Tuna, G. A. Timco, E. J. L. McInnes, R. E. P. Winpenny, Nat. Commun. 2016, 7, 11377; b) A. Gaita-Ariño, F. Luis, S. Hill, E. Coronado, Nat. Chem. 2019, 11, 301-309; c) M. Atzori, R. Sessoli, J. Am. Chem. Soc. 2019, 141, 11339-11352; d) S. von Kugelgen, D. E. Freedman, Science 2019, 366, $1070-1071$.

[6] H. O. Van Oven, H. J. de Liefde Meijer, J. Organomet. Chem. 1969, 19, 373-376.

[7] P. A. Kroon, R. B. Helmholdt, J. Organomet. Chem. 1970, 25, 451-454.

[8] E. Samuel, G. Labauze, D. Vivien, J. Chem. Soc., Dalton Trans. 1979, 956-961.

[9] a) D. Gourier, E. Samuel, J. Am. Chem. Soc. 1987, 109, 4571-4578; b) G. Labauze, J. B. Raynor, E. Samuel, J. Chem. Soc., Dalton Trans. 1980, 2425-2427.

[10] K. A. Lyssenko, M. Y. Antipin, S. Y. Ketkov, Russ. Chem. Bull. 2001, 50, 130-141

[11] H. O. Van Oven, H. J. de Liefde Meijer, J. Organomet. Chem. 1970, 23, 159-163.

[12] J. D. Zeinstra, J. L. De Boer, J. Organomet. Chem. 1973, 54, 207-211.

[13] S. Stoll, A. Schweiger, J. Magn. Reson. 2006, 178, 42-55.

[14] F. Neese, WIREs Comput. Mol. Sci. 2018, 8, e1327.

[15] S. Grimme, J. Chem. Phys. 2006, 124, 034108.

[16] M. Cossi, N. Rega, G. Scalmani, V. Barone, J. Comput. Chem. 2003, 24, 669-681.

[17] M. L. H. Green, D. K. P. Ng, Chem. Rev. 1995, 95, 439-473.

[18] K. J. Standley, R. A. Vaughan, Electron Spin Relaxation Phenomena in Solids, Plenum Press, New York, 1969.

[19] C. J. Yu, M. J. Graham, J. M. Zadrozny, J. Niklas, M. D. Krzyaniak, M. R. Wasielewski, O. G. Poluektov, D. E. Freedman, J. Am. Chem. Soc. 2016, 138, 14678-14685.

[20] K. Bader, D. Dengler, S. Lenz, B. Endeward, S.-D. Jiang, P. Neugebauer, J. van Slageren, Nat. Commun. $2014,5,5304$.

[21] S. S. Eaton, G. R. Eaton, in Biological Magnetic Resonance, Vol. 19 (Eds.: I. J. Berliner, S. S. Eaton, G. R. Eaton), Klewer Academic/ Plenum Publishers, New York, 2000, pp. 29- 154.

[22] a) M. J. Graham, C. J. Yu, M. D. Krzyaniak, M. R. Wasielewski, D. E. Freedman, J. Am. Chem. Soc. 2017, 139, 3196-3201; b) E. R. Canarie, S. M. Jahn, S. Stoll, J. Phys. Chem. Lett. 2020, 3396-3400.

[23] a) T. Yamabayashi, M. Atzori, L. Tesi, G. Cosquer, F. Santanni, M.-E. Boulon, E. Morra, S. Benci, R. Torre, M. Chiesa, L. Sorace, R. Sessoli, M. Yamashita, J. Am. Chem. Soc. 2018, 140, 12090-12101; b) L. Tesi, E. Lucaccini, I. Cimatti, M. Perfetti, M. Mannini, M. Atzori, E. Morra, M. Chiesa, A. Caneschi, L. Sorace, R. Sessoli, Chem. Sci. 2016, 7, 2074-2083; c) M. Atzori, E. Morra, L. Tesi, A. Albino, M. Chiesa, L. Sorace, R. Sessoli, J. Am. Chem. Soc. 2016, 138, 11234-11244.

[24] J. Goffart, L. Hocks, Spectrochim. Acta, Pt. A: Mol. Spectrosc. 1981, 37, 609-614.

[25] A. Albino, S. Benci, L. Tesi, M. Atzori, R. Torre, S. Sanvito, R. Sessoli, A. Lunghi, Inorg. Chem. 2019, 58, $10260-10268$.

[26] a) A. Lunghi, F. Totti, S. Sanvito, R. Sessoli, Chem. Sci. 2017, 8, 6051-6059; b) L. Escalera-Moreno, N. Suaud, A. Gaita-Ariño, E. Coronado, J. Phys. Chem. Lett. 2017, 8, 1695-1700; c) R. Mirzoyan, R. G. Hadt, Phys. Chem. Chem. Phys. 2020.

[27] a) E. Garlatti, L. Tesi, A. Lunghi, M. Atzori, D. J. Voneshen, P. Santini, S. Sanvito, T. Guidi, R. Sessoli, S. Carretta, Nat. Commun. 2020, 11, 1751; b) A. Lunghi, S. Sanvito, Sci. Adv. 2019, 5, eaax7163.

[28] C. Elschenbroich, F. Paganelli, M. Nowotny, B. Neumüller, O. Burghaus, Z. Anorg. Allg. Chem. 2004, 630, $1599-1606$. 
[29] J. J. Liu, J. Mrozek, W. K. Myers, G. A. Timco, R. E. P. Winpenny, B. Kintzel, W. Plass, A. Ardavan, Phys. Rev. Lett. 2019, $122,037202$.

[30] K. Yang, W. Paul, S. H. Phark, P. Willke, Y. Bae, T. Choi, T. Esat, A. Ardavan, A. J. Heinrich, C. P. Lutz, Science 2019, 366, 509-512.

[31] R. Hussain, G. Allodi, A. Chiesa, E. Garlatti, D. Mitcov, A. Konstantatos, K. S. Pedersen, R. De Renzi, S. Piligkos, S. Carretta, J. Am. Chem. Soc. 2018, 140, 9814-9818.

[32] G. Ponti et al., in International Conference on High Performance Computing and Simulation, HPCS 2014, art. no. 6903807, 2014, pp. 1030-1033. 